\title{
Dénutrition Chez Les Patients Cancéreux À L'hôpital National De Niamey (Niger)
}

\section{Mahamane Sani Mahamane Aminou,}

Endocrinologue-Diabétologue-Nutritionniste - Service de Médecine Interne, Hôpital National de Niamey

Assoumane Mariama,

Nutritionniste, Service de Médecine Interne, Hôpital National de Niamey

Ba Mounkaila Halimatou,

Nutritionniste, Centre de recherche spécialisé en Agronomie, Niamey

Malam-Abdou Badé,

Hématologue, Service d'Oncohématologie d'Hôpital National de Niamey

Brah Souleymane,

Daou Mamane,

Médecin Interniste, Service de Médecine Interne,

Hôpital National de Niamey

Balla Abdourahamane,

Agronome, Centre de recherche spécialisé en Agronomie (CRESA)

Mamadou Saidou,

Biologiste, Laboratoire de Reference National pour le VIH de l'hôpital

National Lamorde Niamey

Doi:10.19044/esj.2020.v16n12p359 URL:http://dx.doi.org/10.19044/esj.2020.v16n12p359

\section{Résumé}

Introduction : La dénutrition est un problème fréquemment rencontré chez les patients suivis pour pathologie cancéreuse. Elle est d'origine multifactorielle et constitue un problème important compliquant la prise en charge de ces patients. Méthodologie : Il s'agissait d'une étude prospective et descriptive qui a été menée du 1 juillet au 30 Septembre auprès de patients cancéreux hospitalisés dans le service d'Oncohématologie de l'Hôpital National de Niamey. Le diagnostic de la dénutrition était posé sur la base de l'IMC, l'albuminémie et ou de la perte de poids. Résultats : La prévalence de la dénutrition était estimée à $66,70 \%$. Tous avaient répondu aux critères de l'IMC, 54, 38\% avaient une hypoalbuminémie et $82,40 \%$ avaient perdu du poids. Les tranches d'âge entre 0 et 15 ans et supérieure à 60 ans étaient les plus touchées avec respectivement $76,19 \%$ et $75 \%$. Les cancers à haut risque de dénutrition étaient représentés par les tumeurs de la langue, de l'œsophage 
et les tumeurs pulmonaires. Cette dénutrition était d'origine multifactorielle, d'où l'importance de déterminer les différents facteurs de risque tels que : la nature de la tumeur, la durée d'hospitalisation, les facteurs cliniques (diarrhée, vomissement, anorexie, et douleur). Parmi les patients qui étaient dénutris sous chimiothérapie, $84,61 \%$ étaient dénutris après la troisième (3) cure. Seul l'âge était associé significativement avec la dénutrition $(\mathrm{p}<0,05)$. Sur la prise en charge nutritionnelle, six (6) patients seulement avaient bénéficié d'une alimentation entérale soit $5,9 \%$. Aucun patient dénutri n'avait bénéficié d'une alimentation parentérale.

Mots clés : Dénutrition, cancer, Service Oncohématologie, Niamey

\section{Undernutrition in Cancer Patients at Niamey National Hospital (Niger)}

\section{Mahamane Sani Mahamane Aminou,}

Endocrinologue-Diabétologue-Nutritionniste - Service de Médecine Interne,

Hôpital National de Niamey

Assoumane Mariama,

Nutritionniste, Service de Médecine Interne, Hôpital National de Niamey

Ba Mounkaila Halimatou,

Nutritionniste, Centre de recherche spécialisé en Agronomie, Niamey

Malam-Abdou Badé,

Hématologue, Service d'Oncohématologie d'Hôpital National de Niamey

Brah Souleymane,

Daou Mamane,

Médecin Interniste, Service de Médecine Interne,

Hôpital National de Niamey

Balla Abdourahamane,

Agronome, Centre de recherche spécialisé en Agronomie (CRESA)

Mamadou Saidou,

Biologiste, Laboratoire de Reference National pour le VIH de l'hôpital

National Lamorde Niamey

Abstract

Introduction: Undernutrition is a problem frequently encountered in patients followed for cancer pathology. It is of multifactorial origin and 
constitutes an important problem complicating the management of these patients. Methodology: This was a prospective and descriptive study which was conducted from July 1 to September 30 with cancer patients hospitalized in the Onco-Hematology department of Niamey National Hospital. The diagnosis of undernutrition was made on the basis of BMI, albuminemia and weight loss. Results: The prevalence of undernutrition was estimated at $66.70 \%$. All had met the BMI criteria, 54, 38\% had hypo albuminemia and $82.40 \%$ had lost weight. The age groups between 0 and 15 and over 60 were the most affected with $76.19 \%$ and $75 \%$ respectively. Cancers at high risk of undernutrition were represented by tumors of the tongue, esophagus and lung tumors. This undernutrition was of multifactorial origin, hence the importance of determining the various risk factors such as: the nature of the tumor, the duration of hospitalization, clinical factors (diarrhea, vomiting, anorexia, and pain). Of the patients who were malnourished under chemotherapy, $84.61 \%$ were malnourished after the third (3) course of treatment. Only age was significantly associated with undernutrition $(p<0.05)$. In terms of nutritional management, only six (6) patients had benefited from enteral nutrition, ie $5.9 \%$. No malnourished patient had received parenteral nutrition.

Keywords : Undernutrition, cancer, Onco-Hematology Service, Niamey

\section{Introduction}

La dénutrition est un problème qui ne touche pas uniquement les pays en voie de développement comme certains le pensaient, elle atteint également la population des pays développés souffrant de maladies aiguës ou chroniques, soit les individus en situation économique précaire, les enfants aussi bien que les adultes (Zazzo, 2010).

L'incidence de la dénutrition sur les pathologies est encore insuffisamment considérée, pourtant la dénutrition est souvent un facteur aggravant du pronostic. Combattre une maladie de façon optimale ne peut se faire sans corriger la dénutrition, source de nombreuses complications (Hebuterne, 2006).

La dénutrition est un symptôme fréquemment associé à la pathologie cancéreuse. Elle est observée dans 20 à $70 \%$ des cas (Senesse, 2012). Sa fréquence varie en fonction des études, du type de tumeur, du stade évolutif ainsi que des critères de dénutrition utilisés (Schneider, 2014). La fréquence la plus importante est observée pour les tumeurs du tube digestif proximal $(49,50 \%)$, de la tête et du cou $(45,60 \%)$, ou bien du poumon $(40,20 \%)$. Par contre pour les lymphomes, les sarcomes, les cancers du sein, l'amaigrissement est moins fréquent :-60 à $90 \%$ des patients ne présentent aucune perte pondérale (Senesse, 2012). 
Plusieurs facteurs participent à la dénutrition : une augmentation des dépenses énergétiques due aux perturbations du fonctionnement des cellules et à l'inflammation induite par la tumeur, une réduction des apports alimentaires en raison d'une perte d'appétit (anorexie) liée aux réactions inflammatoires, ou de troubles du goût et de la consommation alimentaire pendant les traitements (NACRE, 2014). D'après Hébuterne et al., (2006) 40 à $80 \%$ des patients sont dénutris en cours de chimiothérapie, radiothérapie ou chirurgie. Par ailleurs trois grands facteurs externes sont susceptibles d'influencer le statut nutritionnel : le traitement, la fréquence de l'alimentation (par ex, lorsque de nombreux examens perturbent le rythme des repas) et les problèmes psychosociaux (Reynders ,2002).

L'absence d'évaluation et de prise en charge peut-être préjudiciable au patient, et principalement l'absence d'évaluation nutritionnelle initiale suggère aussi que, dans un certain nombre de cas, la prise en charge nutritionnelle est inadaptée (Nacre, 2012).

Il n'y a pas encore eu d'études sur le statut nutritionnel des patients cancéreux au Niger, c'est ce qui a motivé la réalisation de cette étude dont le but est d'évaluer l'état nutritionnel des patients atteints de cancers au niveau du service d'Oncohématologie de l'hôpital National de Niamey en vue d'améliorer leur prise en charge nutritionnelle de ses patients

\section{Méthodologie :}

Cette étude prospective transversale s'était déroulée sur 3 mois ( du 1 juillet au 30 Septembre 2015). Il s'agissait d'une étude transversale avec un échantillonnage exhaustif concernant tous les patients hospitalisés dans le service d'oncohématologie de l'hôpital National de Niamey pendant la période étudiée. Etaient inclus dans cette étude, tous les patients hospitalisés dans le service et atteints d'une affection maligne confirmée, traitée ou non par chimiothérapie. Les patients avaient été interrogés et examinés à leurs lits d'hospitalisation et les données portées sur les fiches d'enquêtes. Ils avaient également bénéficié d'un dosage de l'albumine sérique. Les données ont été saisies et analysées à l'aide des logiciels world, Excel et SPSS 22.

\section{Résultats}

\subsection{Les caractéristiques épidémiologiques et cliniques}

Durant cette période, 107 patients hospitalisés pour pathologies malignes ont été colligés. Cinq ont été exclus de l'étude dont, 2 abondons thérapeutiques et 3 décès précoces. En définitif les dossiers de 102 patients ont été exploités. Les tranches d'âge entre 0 et 15 ans et supérieure à 60 ans étaient les plus touchées avec respectivement $76,19 \%$ et $75 \%$. Parmi les patients hospitalisés, 36,20\% avaient été diagnostiqués à l'entrée de cancers digestifs (tumeur du foie, tumeur œsophage ; tumeur gastrique, tumeur langue et 
tumeur palais) et 27,40\% pour cancer hématologique (leucémie et lymphome). Au total 78,40\% de ces patients étaient déjà traités par chimiothérapie.

Le tableau I ci-dessous illustre les différentes sortes de cancers rencontrés dans le service au cours de cette étude :

Tableau I : Répartition des patients selon la nature du cancer

\begin{tabular}{ccc}
\hline Nature du cancer & Effectif & Pourcentage \\
\hline Leucémie & 20 & 19,60 \\
Lymphome & 8 & 7,80 \\
Ostéosarcome & 3 & 2,90 \\
Tumeur cure chevelure & 3 & 2,90 \\
Rétinoblastome & 11 & 10,80 \\
Tumeur sein & 5 & 4,90 \\
Tumeur du Foie & 11 & 10,80 \\
Tumeur Gastrique & 8 & 7,80 \\
Tumeur Langue & 4 & 3,90 \\
Tumeur CEsophage & 9 & 8,80 \\
Tumeur Palais & 5 & 4,90 \\
Tumeur orbitaire & 5 & 4,90 \\
Tumeur pulmonaire & 3 & 2,90 \\
Tumeur vessie & 7 & 6,90 \\
Total & $\mathbf{1 0 2}$ & $\mathbf{1 0 0}$ \\
\hline
\end{tabular}

\subsection{Evaluation de l'état nutritionnel}

Tous les patients avaient été pesés dès leur admission dans le service et le poids des patients était compris entre 45,1 et $60 \mathrm{~kg}$ dans 33,3\% des cas. Le poids moyen était de 45,83 \pm 1,26 avec des extrêmes allant de 8,75 à 83,00 $\mathrm{kg}$.

La perte pondérale a été évalué à 6 mois et 1 mois plus tôt. Il ressort que $82,40 \%$ des patients avaient perdu du poids au cours de notre étude, le poids moyen perdu était de 2,52 $\pm 0,85$ avec des extrêmes allant de 1 à $30 \mathrm{~kg}$. En se basant sur la classification de degré de dénutrition selon 1'IMC, qui permettait de quantifier la maigreur, le tableau $\mathrm{N}^{\circ}$ II permet de conclure que 66,7 patients avaient une dénutrition et se répartissent comme suit : Grade 1 : $14,70 \%$; Grade 2 : $13,80 \%$; Grade 3 : 29,40 \% ; Grade 4 : 7,8 $0 \%$; Grade $5: 1 \%$.

Tableau II : L'état nutritionnel des patients selon les grades de l'IMC (OMS)

\begin{tabular}{lll}
\hline IMC grade & Effectif & Pourcentage \\
\hline Grade 1 & 15 & 14,70 \\
Grade 2 & 14 & 13,80 \\
Grade 3 & 30 & 29,40 \\
Grade 4 & 9 & 8,80 \\
Grade 5 & 0 & 0 \\
Total & $\mathbf{6 8}$ & $\mathbf{6 6 , 7}$
\end{tabular}

Le dosage de l'albumine sérique au niveau du laboratoire avait permis de noter que $52 \%$ avaient une hypoalbuminemie. 
Selon les critères diagnostiques $66,70 \%$ étaient dénutris selon l'IMC, 54,38\% selon le taux d'albuminémie et $29,29 \%$ pour les 2 critères pris à la fois.

\subsection{Facteurs prédictifs de la dénutrition}

\subsubsection{Les signes fonctionnels cliniques}

La figure 1 illustre les différents signes cliniques observés chez les patients. Au total 45,10\% avaient présenté des vomissements.

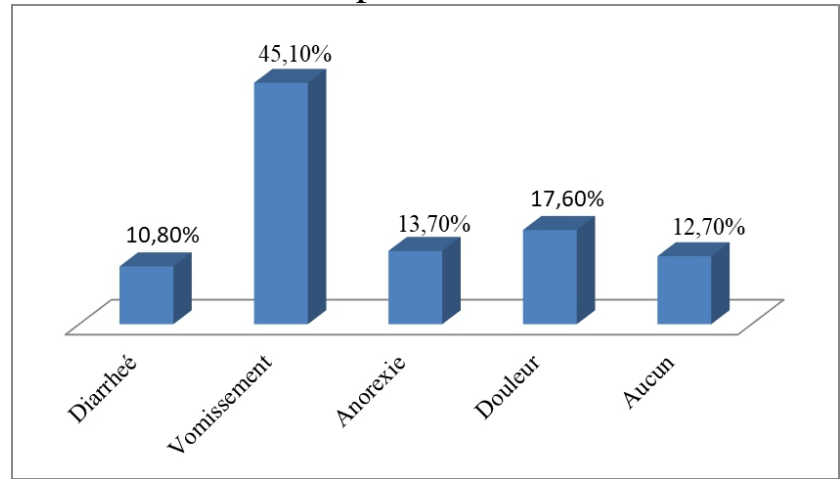

Figure 1 : Répartition des patients selon les signes cliniques

$\checkmark \quad$ La diarrhée : Parmi les 11 patients qui avaient des diarrhées, 8 avaient une malnutrition soit $72,72 \%$ des cas.

$\checkmark$ Les vomissements : Parmi les 46 patients qui avaient présenté des vomissements à l'admission, 33 sont malnutris soit 71,73\% des cas.

$\checkmark \quad$ L'anorexie : Parmi les 14 patients qui avaient une anorexie, 9 étaient malnutris, soit $64,28 \%$ des cas.

$\checkmark$ Syndrome douloureux : Parmi les 18 patients qui présentaient une douleur, 12 étaient malnutris soit $66,66 \%$ des cas.

$\checkmark$ Parmi les 13 patients qui ne présentaient aucun signe ,6 n'étaient pas malnutris, soit $46,15 \%$ des cas.

\subsubsection{Durée d'hospitalisation}

Les patients qui avaient séjourné entre 3 à 15 jours durant leur hospitalisation représentaient $82,30 \%$. Ils étaient suivis par ceux dont la durée de séjour était entre 16 et 30 jours dans 16,7\% des cas.

La durée moyenne d'hospitalisation était de $1,19 \pm 0,41$ jour et des extrêmes allant de 1 à 3 jours.

\subsubsection{Dénutrition en fonction de la nature du cancer}

Le tableau III montre la répartition des patients dénutris en fonction de la nature du cancer : 
Tableau III : Répartition des patients dénutris en fonction de la nature du cancer

\begin{tabular}{ccccc}
\hline Nature du cancer & $\begin{array}{c}\text { Effectif } \\
\text { total }\end{array}$ & $\begin{array}{c}\text { Effectif } \\
\text { dénutris }\end{array}$ & Pourcentage & P-value \\
\hline Leucémie & 20 & 14 & 70 & \\
Lymphome & 8 & 6 & 75 & \\
Ostéosarcome & 3 & 0 & 0 & \\
Tumeur cure chevelure & 3 & 0 & 0 & \\
Rétinoblastome & 11 & 7 & 63,63 & \\
Tumeur du foie & 11 & 6 & 54,54 & \\
Tumeur du sein & 5 & 3 & 60 & \\
Tumeur gastrique & 8 & 6 & 75 & \\
Tumeur langue & 4 & 4 & 100 & \\
Tumeur œsophage & 9 & 9 & 100 & \\
Tumeur orbitaire & 5 & 2 & 40 & \\
Tumeur palais & 5 & 4 & 80 & \\
Tumeur pulmonaire & 3 & 3 & 100 & \\
Tumeur vessie & 7 & 4 & 57,14 & \\
\hline
\end{tabular}

$\mathrm{P}=0,12$

Il n'existe pas de relation statiquement significative entre la nature des tumeurs et la dénutrition. La totalité des patients admis pour tumeur de l'œsophage, pulmonaire et langue étaient tous dénutris.

\section{Discussion}

\subsection{La Fréquence}

La malnutrition, véritable fléau mondial, reste dans les pays en voie de développement un problème de grande acuité. Elle continue d'être l'une des principales causes de mortalité et de morbidité dans le tiers monde. C'est aussi un problème important compliquant la prise en charge des patients atteints de cancer, malgré les progrès au cours des 20 dernières années dans le domaine de l'assistance nutritionnelle (Mears, 1996).

L'estimation de la prévalence de la malnutrition dépend des marqueurs choisis pour la définir et de la population étudiée (type de tumeur, stade évolutif, traitements mis en œuvre, etc...). Chez l'adulte, 30 à $50 \%$ des patients atteints de cancer (hors néoplasies cutanées) étaient amaigris et potentiellement dénutris avant le début du traitement ou pendant le traitement, tous ces chiffres n'ont pas changé depuis 30 ans (Pierre, 2012).

Au cours de cette étude, $67,7 \%$ des patients étaient malnutris selon l'IMC, résultats nettement supérieure à celui trouvé par Laurent (2013) avec16.60 \%. Notre prévalence peut s'expliquer par le fait que les patients consultent tardivement dans les hôpitaux. Ce comportement peut être lié à l'ignorance et ou au manque de moyens financiers.

Les extrêmes d'âge entre 0 et 15 ans étaient plus touchés par la malnutrition avec $76,19 \%$ des cas, résultat supérieure à celui trouvé par Mouchtak (2013) avec 45,1\%. 


\subsection{Facteurs prédictifs de la dénutrition Risque lié aux signes fonctionnels cliniques}

La malnutrition peut être liée à des troubles accompagnants la prise alimentaire, aux nausées et vomissements, aux troubles de transit, ou aux douleurs lors de la mastication, et de la déglutition (stomatites et mucites), ces effets sont le plus souvent induits par les traitements. Les manifestations dépressives ou l'anxiété ou encore la présence de douleurs diverses sont également des facteurs anorexigènes (Pierre, 2012).

La principale cause de dénutrition chez les enfants ayant un cancer était la réduction des apports alimentaires par anorexie. Elle peut exister dès le diagnostic et elle est toujours aggravée au cours du traitement. Dans les pays occidentaux, la dénutrition de l'enfant est principalement liée à la maladie. L'impact des maladies chroniques sur l'état nutritionnel résulte soit des besoins accrus (maladies inflammatoires, etc.), soit des apports insuffisants (affections digestives, anorexie, etc.) ou soit des deux mécanismes (BeauFrère, 1999).

L'anorexie, facteur majeur présent dans 40 à $80 \%$ des cas de dénutrition, peut parfois être psychogène. Elle est le plus souvent liée à des altérations du goût, de l'odorat, avec ou sans candidoses oropharyngées, et aux effets secondaires digestifs communs lors de certaines chimiothérapies (Pressoir, 2010).

Au cours de cette étude 46 patients présentaient des vomissements et 33 étaient malnutris soit 71,73 ce résultat était supérieur à celui publié par Costa en 1991 avec $30 \%$.

Sur 14 patients l'anorexie a était retrouvé chez 9 soit une prévalence de 64,28 $\%$, résultat inferieure à celui de Zazzo et al (2010) avec 78,40\%.

\section{Risques liés à l'hospitalisation}

Les risques liés à l'hospitalisation chez l'enfant comme chez l'adulte est une situation à haut risque nutritionnel. De nombreuses études montrent que la prévalence de la dénutrition reste élevée en milieu hospitalier malgré les progrès considérables réalisés dans ce domaine (Schneider, 2014).

Elle peut être en elle-même la cause d'une diminution des ingesta par le changement d'environnement et des habitudes alimentaires, les horaires de repas imposés, par le jeûne nécessaire pour certains examens complémentaires ou la réalisation des examens ou de la visite au moment des repas selon ZAZZO (2010).

L'alitement prolongé s'ajoute aux autres facteurs et s'accompagne d'une réduction des capacités physiques avec asthénie, puis anorexie et fonte musculaire. La survenue des complications habituelles de décubitus prolongé comme les escarres, favorisées par la malnutrition, est un facteur aggravant de cette dernière (Zazzo, 2010). Chez l'enfant, la dénutrition affecte 15 à $30 \%$ 
des malades, et plus de $65 \%$ des personnes âgées hospitalisées en long séjour en souffrent. Or, la dénutrition au cours d'une hospitalisation ou son aggravation entraîne une augmentation des complications, de la durée moyenne de l'hospitalisation et de la morbidité (Carter, 1983).

Dans cette étude la durée moyenne d'hospitalisation pour tous les patients était de 1.19 jours, inferieure à celui trouvé par Laurent : 3.02 jours (Laurent, 2013.). Le constat est que plus la durée est longue plus on a un risque de dénutrition élevée et le seul patient qui a été hospitalisée pendant plus de 30 jours était sévèrement dénutri.

\section{Risques liés à la tumeur}

Chez l'enfant, la malnutrition semble résulter plus rarement que chez 1. Le type de la tumeur semble en cause, alors que l'état nutritionnel des enfants porteurs de certaines tumeurs malignes au diagnostic n'est pas différent de celui des enfants avec tumeurs bénignes. La prévalence de la dénutrition au diagnostic semble plus élevée chez les enfants atteints de sarcome d'Ewing que pour les autres types de tumeurs (Mears, 1996).

Le type de tumeur pourrait être lui aussi un facteur de risque de dénutrition d'après la littérature, les sarcomes d'Ewing, les neuroblastes, les gliomes diencéphaliques, les localisations ORL, les cancers métastatiques et les ostéosarcomes sont fortement suspects d'être à haut risque de dénutrition celle-ci peut être due soit à la tumeur elle-même (cachexie diencéphalique, tumeur ORL par obstacle mécanique), soit à l'augmentation de la dépense énergétique, soit aux conséquences des traitements (troubles digestifs, troubles buccaux,). (Carter, 1983 \& Van Eys, 1979:).

D'après Laurent, la fréquence de la dénutrition est variable selon la localisation tumorale : œsophage : $61 \%$, estomac : $59 \%$ foie : $37 \%$ inferieure (Laurent, 2013), à nos résultats : tumeur œsophage (100\% des cas), les tumeurs gastriques (75\% des cas), et tumeur du foie $(54,54 \%)$.

\section{Risques liés au traitement}

Pour les enfants traités par chimiothérapie, il est difficile de distinguer la part du risque lié à la gravité de la maladie, et la part liée aux effets secondaires du traitement. Cependant, que ce soit pour la première ou la seconde raison, ces enfants sont plus vulnérables que les autres et il est raisonnable de vouloir les prendre en charge d'un point de vue nutritionnel dès leur admission (Zazzo, 2010).

Dans cette série, $70 \%$ des patients qui étaient sous chimiothérapie étaient dénutris résultat supérieure à celui de Mouchtak : 59,5\% (Mouchtak, 2013). L'étude a révélé que $84,61 \%$ de nos patients étaient dénutris après 3 cures supérieures à celui de Chermiti(2013) et coll qui avaient trouvé après trois cures de chimiothérapie $77 \%$ cas . 


\section{Conclusion}

La dénutrition est fréquente chez les patients cancéreux. Il avait été constaté que $66,70 \%$ étaient dénutris qu'ils soient sous chimiothérapie ou non. Les cancers le plus à risque de dénutrition étaient les cancers digestifs et pulmonaires. Plusieurs facteurs associés (Age, durée hospitalisation, nature de la tumeur ...) à la dénutrition étaient retrouvé mais non significatifs à part l'âge. Dans le cadre de la prise en charge du patient atteint de cancer, l'évaluation nutritionnelle et le suivi diététique doivent donc impérativement faire partie intégrante du suivi et du traitement, au même titre que la prise en charge psychologique et antalgique.

Des recherches supplémentaires sont nécessaires pour analyser l'impact de la nutrition sur la morbidité, la mortalité et la qualité de vie chez ces patients. D'autres études doivent être initiées pour approfondir le sujet notamment concernant la prévalence globale de la dénutrition chez les patients cancéreux, y compris ceux de la chirurgie ; ou encore élargir l'étude à tous les services concernés avec comme critères l'IMC et ou l'albuminémie et ou la perte de poids, afin d'apprécier l'ampleur globale de la dénutrition chez les personnes atteintes de cancer.

\section{References:}

1. Beau-frere B, Birge J, Burlet, Campillo B, Couet C, Fouque D. (1999). Dénutrition de L'Enfant. Éditions Inserm : 163-76.

2. Carter P, Carr D, Van eys J. (1983). Nutritional parameters in children with cancer. J Am Diet Assoc; 82:616-22.

3. Chermiti Ben Abdallah F, Ben Saïd H, Chamkhi N, Ferchichi M, Chtourou A, Taktak S et all. (2013). Evaluation de l'état nutritionnel des patients atteints de cancer bronchopulmonaire primitif. La tunisie médicale ; 91 (010) : 600-604.

4. Costa G. et Donaldson S.S. (1979). Current concepts in cancer: effects of cancer and cancer treatment on the nutrition of the host. $\mathrm{N}$ Engl J Med ;300 :1471-4.

5. Hébuterne X, Lemarié E, Michallet M, Beauvillain de Montreuil C, Goldwasser F. Prévalence de la dénutrition au cours du cancer : une enquête nationale un jour donné. Nutrition Clinique et Métabolisme ; 20 : S86.

6. Laurent G. (2013). Evaluation de 1 'état nutritionnel des enfants atteints de cancers suivis en oncologie pédiatrique, Thèse $\mathrm{N}^{\circ} 4913$, Grenoble, p103.

7. Mears E. (1996). "Outcomes of continuous process improvement of a nutritional care program incorporating serum prealbumin measurements." Nutrition, $12: 479-84$. 
8. Mouchtak NA. (2013). Evaluation de l'état nutritionnel des enfants atteints de cancer suivis en oncologie pédiatrique. Thèse $\mathrm{N}^{\circ} 04913$, Maroc, 116p.

9. Nacre (2012). Nutrition chez le patient adulte atteint de cancer, $24 \mathrm{p}$.

10. Pierre S, Patrick B. et René Jean B. (2012). Oncology nutrition guidelines SFNEP. Nutrition chez le patient atteint de cancer , 84p.

11. Pressoir M, Desné S, Berchery D, Rossignol G, Poiree B, Meslier M et all .(2010). Prevalence, risk factors and clinical implications of malnutrition in French Comprehensive Cancer Centers. Br J Cancer, 102(6): 966-71.

12. Reynders L, Van Loon MR, Wellens C, Winter E, Fondation contre le Cancer (2002). Les principaux problèmes alimentaires du patient en oncologie. GUIDE ET CONSEILS PRATIQUES https://www.cancer.be/sites/default/files/guide-oncodieteticien: 66p.

13. Schneider J-M, (2014). Dénutrition, in Schlienger J-L. Nutrition clinique pratique chez l'adulte et l'enfant. Elsevier Masson, 2eme édition : 121-39

14. Senesse P, Vasson $M-P$ (2012). Nutrition chez le patient adulte atteint de cancer : quand et comment évaluer l'état nutritionnel d'un malade atteint de cancer Comment faire le diagnostic de dénutrition et le diagnostic de dénutrition sévère chez un malade atteint de cancer ? Quelles sont les situations les plus à risque de dénutrition? /Nutrition clinique et métabolisme, $26: 165-188$

15. Van eys J. (1979). Malnutrition in children with cancer: incidence and consequence. Cancer, 43(5): 2030- 5.

16. Zazzo J. F, Antoun S, Basdevant A et al. (2010). Ministère de la santé et des sports, Société française Nutrition. Dénutrition une pathologie méconnue en société d'abondance: programme national nutrition sante 2010. Société française Nutrition clinique et métabolisme www.sante.gouv.fr/htm/pointsur/nutrition/actions42.htm 